\title{
Reduced expression of TLR4 is associated with the metastatic status of human colorectal cancer
}

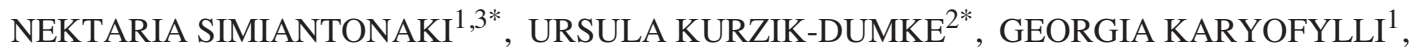 \\ CAREN JAYASINGHE ${ }^{1,3}$, ROMI MICHEL-SCHMIDT ${ }^{1}$ and CHARLES JAMES KIRKPATRICK ${ }^{1}$ \\ ${ }^{1}$ Institute of Pathology, Johannes Gutenberg University, Langenbeckstrasse 1, 55131 Mainz; ${ }^{2}$ Institute of Medical \\ Microbiology and Hygiene, Comparative Tumor Biology Group, Johannes Gutenberg University, \\ Obere Zahlbacher Strasse 63, 55131 Mainz, Germany
}

Received February 16, 2007; Accepted March 19, 2007

\begin{abstract}
Signaling mediating colorectal cancer (CRC) progression is incompletely understood. Previously, we identified lipopolysaccharide (LPS), an endotoxin of ubiquitously existing colonic bacteria, as a pivotal stimulus increasing the metastatic potential of human CRC. Since the ubiquitous colonic bacteria release large amounts of LPS this observation could be of enormous relevance for the progression of CRC. In this study we present data contributing to the elucidation of its mode of action. Since both receptors CD14 and TLR4 act as LPS mediators, we determined their expression in various CRC cell lines and in 115 nonmetastatic, lymphogenous-metastatic and haematogenousmetastatic CRC specimens. Here we showed that CD14 was not expressed in normal colon epithelium, in non-metastatic and metastatic CRC. Furthermore, we showed that diverse CRC cell lines did not express CD14 under normal conditions and after LPS stimulation. Thus, CD14 can be ruled out as a mediator of LPS-induced signaling related to CRC progression. In contrast, we found that normal colon epithelium and CRC cell lines were positive for TLR4. Furthermore, both lymphogenous and haematogenous metastatic cases showed either loss of expression or strong downregulation of TLR4 as compared to normal tissue and to non-metastatic tumors. We
\end{abstract}

Correspondence to: Dr N. Simiantonaki, ${ }^{3}$ Present address: Institute of Pathology Klinikum Leverkusen, Gesundheitspark 11, 51137 Leverkusen, Germany

E-mail: simiantonaki@klinikum-lev.de

Professor U. Kurzik-Dumke, Institute of Medical Microbiology and Hygiene, Comparative Tumor Biology Group, Johannes Gutenberg University, Obere Zahlbacher Strasse 64, 55131 Mainz, Germany

E-mail:kurzik@mail.uni-mainz.de

${ }^{*}$ Contributed equally

Key words: TLR4, CD14, lipopolysaccharide, colorectal cancer found that LPS stimulation resulted in significant TLR4 upregulation in cells expressing lower constitutive TLR4 levels such as $\mathrm{CaCo}$, whereas no significant response to LPS was observed in cells characterized by relatively high amounts of constitutive TLR4. Our data suggest that TLR4 expression may be associated with mechanisms preventing CRC progression.

\section{Introduction}

Colorectal cancer (CRC) is the third most commonly diagnosed cancer in the world and the fourth most frequent cause of cancer-related deaths which is primarily due to metastases (1). These epidemiological data show that these tumors are a major public health problem and emphasize the importance of elucidation of the mechanisms inducing their progression.

Histological examination of CRC shows immunoinflammatory cells in the vicinity of these tumors. This topographical arrangement implies a direct relationship between inflammation, immune response and cancer. The processes in question are strongly interconnected and build a dynamic system. The interactive communication between the components of this system is controlled by the tumor microenvironment (2). Both the tumor cells and the peritumoral immuno-inflammatory cells produce mediators, cytokines activating the immune system and controlling contact with the endothelium (3-5). Generally, cytokines play a dual, and more interestingly, an opposing role in tumorigenesis. They drive malignant progression and, thus, the metastatic potential of the tumor. On the other hand they can also act as antitumor agents (6).

The synthesis and secretion of some of the cytokines of importance in host immune and inflammatory cells can be induced by the endotoxin of gram-negative bacteria e.g. E. coli and LPS (7). In previous studies we identified LPS as a pivotal stimulus leading to endothelial activation in distinct tumors, among them CRC $(8,9)$. Since the ubiquitous colonic bacteria release large amounts of lipopolysaccharide (LPS) this observation is of enormous relevance for the progression of CRC. Generally, it has been postulated that cells utilize different ways to recognize LPS and in consequence that LPS 
may induce a variety of cellular responses $(10,11)$. CD14, a glycosylphosphatidylinositol (GPI)-anchored protein is one of the major receptors which recognizes and binds LPS (12). Monocytes/macrophages, neutrophils and nonmyeloid cells such as endothelia and gingival fibroblasts have been identified as positive for CD14 (13-15). Its expression in epithelia, among them the cells of the gastrointestinal tract, has been controversially discussed (16-19). Since CD14 lacks a transmembrane-spanning region it cannot directly transmit a signal (10). Further molecules are considered to be involved in CD14-mediated signal transmission.

Experimental evidence exists that the TLR4 receptor can transduce LPS signals leading to activation of multiple signaling molecules, such as MAPK, JNK/SAPK, p38 and $\mathrm{NF}-\kappa \mathrm{B}$ (11). The latter induces the transcription of the genes encoding cytokines, chemokines and adhesion molecules crucial to both the initiation of the inflammatory response and the recruitment of immune competent cells (20). The participation of TLR4 in the aforementioned signal cascade implies that this receptor may play an important role in the control of metastasis. Data supporting this hypothesis have been provided from in vivo studies performed using TLR4deficient mice. Whereas TLR4-competent mice showed inhibition of tumor growth and lung metastasis, TLR4deficient mice did not show this protective effect (21).

TLR4 is predominantly expressed in monocytes/ macrophages and polymorphonuclear leukocytes. However, endothelia and epithelial cells are also positive for this receptor $(19,22,23)$. With regard to TLR4 expression in colorectal epithelia, extensive studies have been performed using inflammatory tissue $(19,24-26)$. They have provided evidence that TLR4 serves as a critical link between toxic compounds in the intestinal flora and the mucosal immune system. Studies concerning its role in the events leading to progression of CRC have not yet been published.

In the context of the identification of LPS as a potent activator for the contact of tumor cells with the endothelium as described previously we considered it an important agent mediating tumor progression $(8,9)$. In this context we asked the question whether LPS recognition in colon epithelium occurs via the CD14 and/or the TLR4 receptor. We determined the expression patterns of both molecules, CD14 and TLR4, in various CRC cell lines and in a series of nonmetastatic, lymphogenous-metastatic and haematogenousmetastatic CRC using techniques such as reverse transcription (RT)-PCR, Western blot analysis and immunohistochemistry. Interestingly enough, our results showed that both the CRC cell lines and the in situ tumors investigated expressed TLR4 but not CD14. We found that reduced expression of TLR4 was a frequent occurrence in metastatic carcinomas. Thus, our data suggest that TLR4 may be involved in processes inhibiting metastasis, thus confirming in the human the conclusions drawn from murine knockout models.

\section{Materials and methods}

Cell culture conditions and LPS stimulation experiments. The human CRC cell lines SW837, HRT18, CX-1, CX-2, SW620, SW948, HT-29 and CaCo2 were grown in RPMI-
1640 medium supplemented with Glutamax (Sigma), 10\% heat-deactivated FCS (Gibco), $1 \%$ penicillin (Gibco) and $1 \%$ streptomycin (Gibco) at $37^{\circ} \mathrm{C}$ with $5 \% \mathrm{CO}_{2}$. To induce the differentiation process and CD14 expression in the U937 cells used as positive control for CD14 expression, the culture medium was additionally supplemented with PMA $(10 \mathrm{ng} / \mathrm{ml})$ (27). LPS stimulation was performed by replacing the growth medium of subconfluent cultures with medium supplemented with LPS derived from E. coli (Sigma), $1 \mu \mathrm{g}$ / $\mathrm{ml}$, for $4 \mathrm{~h}$. After stimulation cells were harvested and used for expression analysis.

Tissue samples. Colorectal tissue samples were obtained from 115 patients undergoing elective surgery for CRC at the University of Mainz during the years 1995-1999. The morphological classification of the carcinomas was conducted according to WHO specifications. All tumors were staged following the guidelines of the TNM Classification of Malignant Tumors (1997) (28). With respect to the T status all investigated tumors were T3 and moderately differentiated (G2). With regard to the metastatic potential the cases investigated were separated into three groups. The first group encompassed 40 cases without tumor metastasis to regional lymph nodes or distant organs (N0/M0). Seventy-five cases metastasized whereas 40 of the cases were characterized by haematogenous $\left(\mathrm{M}^{+}\right)$and 35 by lymphogenous metastases $\left(\mathrm{N}^{+}\right)$. In each case follow-up data were obtained from hospital charts and through correspondence with the physicians in charge during a period of 5 years after surgery.

Human placenta and spleen sections were used in control experiments for immunohistochemistry.

Antibodies. Primary antibodies used included goat polyclonal anti-TLR4 (H-80, Santa Cruz Biotechnology, Inc.), mouse monoclonal anti-CD14 (LOXO), mouse monoclonal antiCD14 (UCM-M1, Santa Cruz Biotechnology, Inc.) and rabbit polyclonal anti- $\beta$-actin (Serva). Secondary antibodies included horse anti-mouse biotinylated IgG (Vector) and rabbit anti-goat biotinylated $\operatorname{IgG}$ (Vector).

RNA isolation and semiquantitative reverse transcription$P C R$. RNA isolation was performed using the RNeasy kit (Qiagen, Hilden, Germany) in accordance with the manufacturer's protocols. Actin and CD14 transcripts were analyzed by RT-PCR. RT was performed with the help of the Omniscript RT kit (Qiagen) in accordance with the manufacturer's manual. As a template for the specific PCR reactions $2 \mu \mathrm{l}$ of the synthesized cDNA pool was used. To amplify the $\beta$-actin-specific fragment, 574 base pair (bp), the primers 5'-GAC CTG ACT GAC TAC CTC ATG A-3' (forward) and 5'-AGC ATT TGC GGT GGA CGA TGG AG-3' (reverse) were used. Amplification of the human CD14specific fragment, $372 \mathrm{bp}$, was performed using the primers 5'-GCT GTG TAG GAA AGA AGC TA-3' (forward) and 5'TTT AGA AAC GGC TCT AGG TTG-3' (reverse). Amplification reactions were performed with the help of the Gene amplification PCR system 2400 (Perkin Elmer) thermocycler. The PCR products were separated on agarose gels $(2 \%)$ supplemented with ethidium bromide and analyzed by viewing under UV. 
Western blot analysis. Protein extracts from harvested human cells and normal and tumor epithelia derived from patients undergoing surgical resection of CRC were prepared in TKM buffer (50 mM Tris, pH 7.5, $150 \mathrm{mM} \mathrm{KCl}$ and $5 \mathrm{mM} \mathrm{MgCl}_{2}$ ) using a Dounce homogenizer. The total protein content was determined using the Bio-Rad protein assay (Bio-Rad Laboratories $\mathrm{GmbH}$ ). For Western blotting aliquots containing $20 \mu \mathrm{g}$ of total protein from each sample were separated on sodium dodecyl sulphate-polyacrylamide gels SDS-PA (10\%) and then transferred to polyvinylfluoride (PVDF) membranes (Immobilen-P, Millipore Corp.) in accordance with standard procedures. Incubation with the primary antibodies to TLR4, CD-14 and $B$-actin was conducted overnight at $4^{\circ} \mathrm{C}$. Immunodetection was performed using the alkaline phosphatase (AP)-conjugated anti-mouse and anti-rabbit IgG (Sigma). AP was detected using a mix containing $0.45 \%$ nitroblue tetrazolium (NBT, Serva) and $0.35 \%$ bromo-4-chloro-3-indolyl phosphate toluidinium salt (X-Phosphat, Serva) in AP buffer (100 mM NaCl, $50 \mathrm{mM}$ $\mathrm{MgCl}_{2}$ and $100 \mathrm{mM}$ Tris, $\mathrm{pH}$ 9.2). Primary antibodies were used in a 1:100 dilution. Secondary antibodies were used at concentrations suggested by the suppliers.

Immunohistochemistry. All immunohistochemical reactions were conducted using formalin-fixed and paraffin-embedded samples. After deparaffinization the samples were treated in a microwave oven in EDTA buffer for $15 \mathrm{~min}$. Incubation with the primary antibodies to TLR 4 and CD14, and the secondary antibodies, horse anti-mouse biotinylated IgG (for CD14) and rabbit anti-goat biotinylated IgG (for TLR4) were carried out in accordance with standard protocol using the Vectastain Elite reagent (Vector Laboratories, Inc.). AntiTLR4 was used at the concentration 1:200, both anti-CD14 antibodies were used at the concentration of $1: 100$ and all secondary antibodies at the a concentration of 1:200. Sections were counterstained with Mayer's hematoxylin. To prove the specificity of the immunoreactions every CRC sample $(n=115)$ was stained solely with the secondary antibody. Control reactions were performed for each of the primary antibodies used. As positive control for CD14 staining formalin-fixed and paraffin-embedded human placenta was used as proposed by the antibody supplier. As positive control for TLR4 immunoreactivity, human spleen was used. Immunostaining reactions of each sample were evaluated by three authors independently (N.S., C.J., G.K.) without knowing the clinicopathological findings. The evaluation of the immunohistochemical staining was performed in accordance with a previously established scoring method classifying the tumors into four groups with respect to the staining intensity; negative, weak, moderate, and strong staining. In those cases where heterogeneous staining was observed within the same sample, the level of staining which was visible in $>50 \%$ of the cells was chosen for the classification into a defined group. In those cases where the evaluation results of the three independent authors were different, the specimens were re-evaluated.

Statistics. The association of staining intensity with tumor stadium was assessed with the $\chi^{2}$ (Fisher's exact test). $\mathrm{p}<0.05$ was considered to be significant in all statistical analyses.
(A)

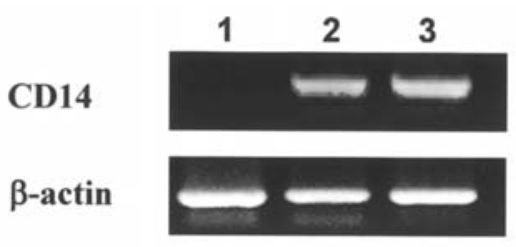

(B)

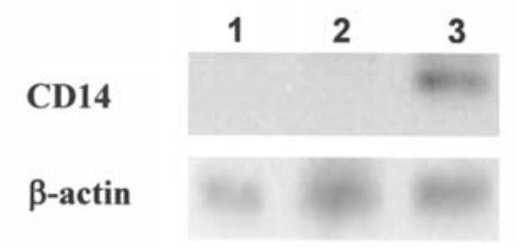

Figure 1. (A) RT-PCR analysis of CD14 expression in the non-stimulated (lane 1) and LPS-stimulated (lane 2) $\mathrm{CaCo} 2$ cells, and in the CD14-positive monocytic differentiated U937 cells (control, lane 3). To detect CD14 a 372-bp fragment, corresponding to the CD14 cDNA was amplified. The quality of the reaction was judged upon amplification of a specific $\beta$-actin fragment, 574 bp in size. (B) Immunodetection of CD14 in non-stimulated (lane 1) and LPS-stimulated (lane 2) $\mathrm{CaCo} 2$ cells, and in U937 cells (lane 3). Note the induction of CD14 expression in $\mathrm{CaCo} 2$ cells after LPStreatment. Detection of $\beta$-actin was performed as a control for loading.

\section{Results}

CD14 is neither expressed in normal human colon epithelium nor in CRC and their derived cell lines. LPS has been found to induce intracellular signaling via the CD14 receptor, which is primarily expressed on inflammatory cells such as monocytes/macrophages and on polymorphonuclear and nonmyeloid cells (12-15). Reports concerning its expression on epithelial cells are controversial (16-19). To ascertain whether in epithelial cells LPS-induced cellular responses can be maintained via CD14, we first looked for its expression in diverse CRC cell lines: SW837, HRT18, CX-1, CX-2, SW620, SW948, HT-29 and CaCo2 using RT-PCR and Western blotting. Furthermore, we determined its inducibility after LPS stimulation. CD14 transcription was detected by RT-PCR of a 372-bp-specific fragment corresponding to the CD14 gene (Fig. 1A). As a positive control for CD14 expression the cell line U937 was used (27). All cell lines used in this study were negative for the CD14 transcript under normal conditions (not shown). LPS stimulation resulted in induction of $\mathrm{CD} 14$ expression solely in the $\mathrm{CaCo} 2$ cells (Fig. 1A, lane 2), while all further investigated cell lines remained negative. For the non-stimulated and LPS-stimulated cells: SW837, HRT18, CX-1, CX-2, SW620, SW948 and HT-29, the data compiled by Western blotting were congruent with the results obtained by RT-PCR (not shown). Notably, in the case of the $\mathrm{CaCo} 2$ cells LPS stimulation resulted in CD14 transcription but not translation (Fig. 1B, lane 2). Thus, the translation of the LPS-induced CD14 transcript was prevented by an as yet unknown mechanism. This topic will be considered in future analysis and, thus, will not be raised any further in this study.

To corroborate in situ the results obtained in vitro we investigated the CD14 expression profiles in normal colon 


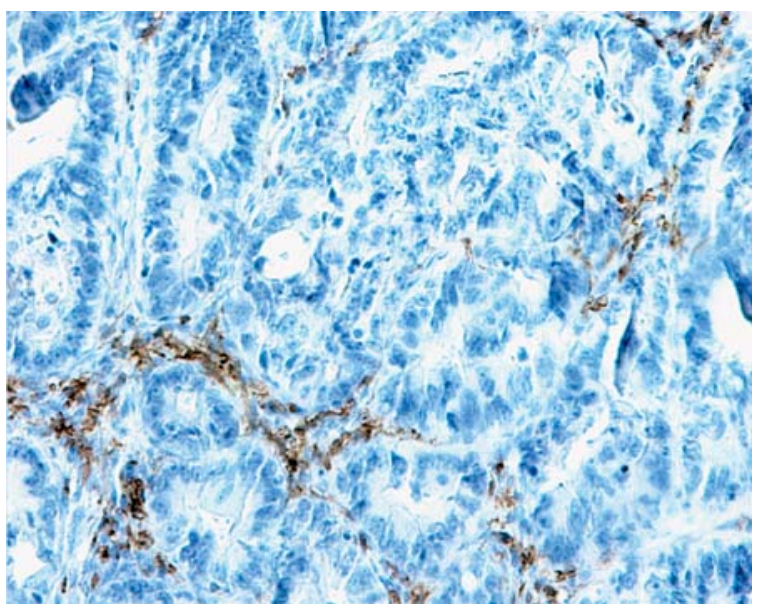

Figure 2. CD14 expression in primary CRC. Note the strong CD14 staining of the inflammatory cells in contrast to the clearly negative tumor epithelium. For staining, the mouse monoclonal anti-CD14 antibody (Santa Cruz) was used. The section was counterstained with Mayer's hematoxylin. Magnification, $\mathrm{x} 400$.

epithelium, in 6 non-metastatic (N0/M0) and 8 metastatic $\left(\mathrm{N}^{+} / \mathrm{M}^{+}\right)$CRC by Western blotting (not shown) and by immunohistochemistry (Fig. 2). Notably, all investigated cases were negative for CD14. Thus, this analysis confirmed the results as shown for the cell lines SW837, HRT18, CX-1, CX-2, SW620, SW948, and HT-29. Since the detection was performed using two monoclonal antibodies against CD14, non-specificity of the reaction could be excluded. The latter was further supported by the positive CD14 stain of the peritumoral inflammatory cells shown exemplarily in Fig. 2.

LPS induces TLR4 expression in the well-differentiated $\mathrm{CaCo} 2$ cells. The in vitro study described above clearly indicated that CD14 was not expressed in the CRC cell lines. Thus, here, the action of LPS must occur via a different path. Since TLR4 has also been postulated to mediate LPS signaling we asked ourselves whether it could act as a receptor for LPS in colorectal cells $(10,11)$. In that context we investigated TLR4 expression in the above CRC cell lines by Western blotting and immunohistochemistry, similarly as described above for CD14. As shown in Fig. 3 all eight cell lines were positive for TLR4. However, its expression level differed from very weak in the well-differentiated $\mathrm{CaCo} 2$ cells to moderate or high in the remaining cells. To ascertain whether TLR4 can be considered a potential LPS ligand we next examined whether the treatment of the cells with the latter influences TLR4 expression. Notably, LPS treatment of the $\mathrm{CaCo} 2$ cells, characterized by a lower native TLR4 level, resulted in a drastic increase in TLR4 concen-tration (Fig. 3). In contrast, no significant response to LPS was observed in cell lines characterized by high amounts of native TLR4.

TLR4 is differentially expressed in human CRC. The upregulation of TLR 4 in $\mathrm{CaCo} 2$ cells after LPS treatment as described above suggested to us that under some circumstances this receptor may mediate LPS function(s). This novel finding is of great importance in the context of the involvement of TLR4-mediated signaling in tumor progression. To approach this subject we investigated the expression profile of TLR4 in primary colon carcinomas by Western blotting (Fig. 4) and immunohistochemistry (Fig. 5). Fourteen cases, 6 nonmetastatic (N0/M0) (Fig. 4A) and 8 metastatic $\left(\mathrm{N}^{+} / \mathrm{M}^{+}\right)$ (Fig. 4B), were examined by Western blot analysis. All specimens were derived exclusively from the surface of the tumor. Furthermore, they were comparable with regard to the amount of the tumor cells and the adjacent tissue including the inflammatory cells positive for TLR4. As shown in Fig. 4A, in all 6 non-metastatic cases TLR4 was detected in the tumor. In 4 cases (1-4), its expression level was similar in the tumor and in the corresponding non-neoplastic tissue. In two cases (5 and 6), TLR4 was upregulated in the epithelium of the tumor. Among the metastatic cases (Fig. 4B) different TLR4 expression profiles were found. In four cases (1-4) TLR4 was detected in the normal tissue, whereas the tumor was either negative or characterized by a very low expression level of the receptor. In case 5 TLR4 was not found in either the normal or in the tumor epithelium. In two cases ( 6 and 7 ) its expression level was similar in both the normal and the tumor epithelium. In contrast in case 8, TLR4 was expressed in the tumor, but not in the normal epithelium. Generally, the expression profiles detected were heterogenous with respect to the level of TLR4 in both the non-metastatic and metastatic

$\begin{array}{llllll}\text { SW837 HRT18 CX-1 CX-2 } & \text { SW620 } & \text { SW948 } & \text { HT-29 } & \text { CaCo2 }\end{array}$
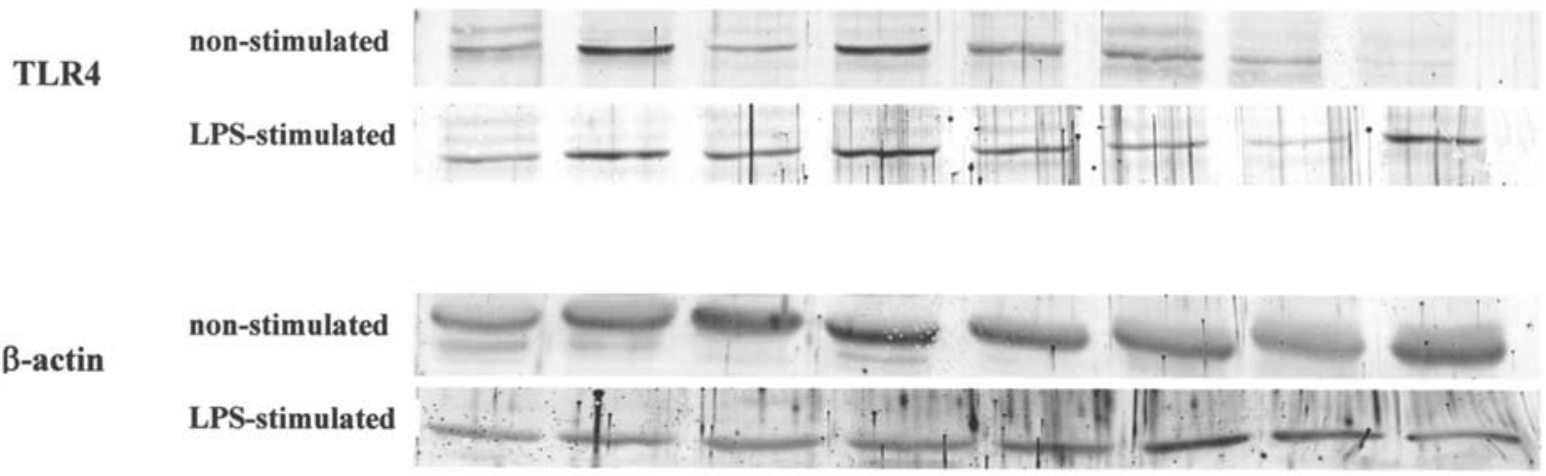

Figure 3. Detection of TLR4 in non-stimulated and LPS-stimulated human CRC cell lines by Western blotting. Immunodetection was performed using the anti-TLR4 antibody (Santa Cruz). Staining with anti-ß-actin was performed as a control for loading. 
(A)

\begin{tabular}{|c|c|c|c|c|c|c|c|c|c|c|c|}
\hline & & & 2 & 3 & & 4 & & 5 & & 6 & \\
\hline $\mathrm{N}$ & $\mathrm{T}$ & $\mathrm{N}$ & $\mathrm{T}$ & $\mathrm{N}$ & $\mathrm{T}$ & $\mathrm{N}$ & $\mathrm{T}$ & $\mathrm{N}$ & $\mathrm{T}$ & $\mathrm{N}$ & $\mathrm{T}$ \\
\hline- & - & $=$ & - & $=$ & - & - & $=$ & & - & & 4 \\
\hline- & {[} & & - & $\square$ & & & $=0$ & & 3 & -1 & - \\
\hline
\end{tabular}

(B)

\begin{tabular}{|c|c|c|c|c|c|c|c|c|c|c|c|c|c|c|c|}
\hline & 1 & & 2 & & & & 4 & . & ; & $\theta$ & 5 & 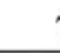 & 7 & 8 & \\
\hline $\mathrm{N}$ & $\mathrm{T}$ & $\mathrm{N}$ & $\mathrm{T}$ & $\mathrm{N}$ & $\mathrm{T}$ & $\mathrm{N}$ & $\mathrm{T}$ & $\mathrm{N}$ & $\mathrm{T}$ & $\mathrm{N}$ & $\mathrm{T}$ & $\mathrm{N}$ & $\mathrm{T}$ & $\mathrm{N}$ & $\mathrm{T}$ \\
\hline سع & & 二 & Ti & - & & & 4 & & & - & 4 & - & \pm & & +4 \\
\hline$=$ & & $\theta$ & - & & - & 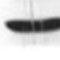 & & - & - & - & - & - & - & - & $\rightarrow$ \\
\hline
\end{tabular}

Figure 4. TLR4 detection in 14 human primary CRC. Regarding the metastatic status, the cases investigated were separated into non-metastatic (A) and metastatic (B) tumors. For immunodetection the anti-TLR4 (Santa Cruz) was used. Staining with anti-ß-actin (bottom blots respectively) was performed as a control for loading. N, normal epithelium; $\mathrm{T}$, tumor epithelium.

Table I. Distribution of non-metastatic, lymphogenous-metastatic and haematogenous-metastatic CRC according to TLR4 expression level.

\begin{tabular}{lccccc}
\hline \multirow{2}{*}{ CRC } & & \multicolumn{4}{c}{ TLR4 expression } \\
\cline { 4 - 6 } & $\mathrm{n}$ & Negative & Weak & Moderate & Strong \\
\hline Non-metastatic & 40 & $0(0 \%)$ & $7(17 \%)$ & $17(43 \%)$ & $16(40 \%)$ \\
Lymphogenous-metastatic & 35 & $4(11 \%)$ & $9(26 \%)$ & $13(37 \%)$ & $9(26 \%)$ \\
Haematogenous-metastatic & 40 & $7(18 \%)$ & $10(25 \%)$ & $14(35 \%)$ & $9(22 \%)$ \\
\hline
\end{tabular}

$\mathrm{n}$, number of investigated cases.

tumors. However, whereas all non-metastatic tumors expressed TLR4, in most metastatic tumors either loss of expression or downregulation was observed.

To verify the result obtained by Western blotting, a large pool of 115 CRC was investigated by immunohistochemistry (Table I). One hundred and four cases $(90 \%)$ were proven positive for TLR4 on the cell membrane and the cytoplasm of the tumor epithelia. With regard to the metastatic status the cases investigated represented three classes. Among the 40 first class cases no evidence of tumor metastasis to regional lymph nodes or distant organs was found during a five-year follow-up. The remaining 75 cases metastasized. Forty specimens were characterized by haematogenous and 35 by lymphogenous metastases. With respect to the $\mathrm{T}$ status all tumors investigated were T3 and moderately differentiated (G2). The latter selection was performed with respect to the relationship postulated above between the expression level of TLR4 and the metastatic status of the tumor. As shown in Table I the investigated samples showed heterogenous TLR4 expression independently of their metastatic status. Among the positive tumors weak, moderate and strong expression levels were distinguished (Table I, Fig. 5). If we choose moderate/strong immunoreactivity as the cut-off value for the assignment of TLR4 positivity, a conspicuous tendency is visible, namely, increased metastatic status of the tumor correlates with loss of TLR4. Whereas $17 \%$ of the nonmetastatic tumors were TLR4 negative, among the lymphogenous- and haematogenous-metastatic cases, 37 and $43 \%$ were found, respectively. The results were just below the statistical significance level in the lymphogenous $(\mathrm{p}=0.06)$ and significant in the haematogenous $(\mathrm{p}=0.027)$ metastatic tumors.

TLR4 expression in CRC does not correlate with peritumoral inflammation. CRC frequently shows an inflammatory infiltration of the peritumoral stroma. The degree of inflammation varies from mild, characterized by minimal to moderate lymphoplasmatic infiltrates accompanied by a sparse neutrophilic component, to severe, characterized by dense confluent mixed inflammatory infiltrates with lymphoid follicles and occasional abscesses. To prove whether a relationship exists between the metastatic state, the TLR4 expression and the surrounding inflammation, we examined the grade of the peritumoral inflammation of the 115 carcinomas discussed above (Table II). Interestingly enough, the degree of the inflammatory infiltration was fairly uniform in the nonmetastatic and lymphogenous-metastatic carcinomas. In both groups $\sim 60 \%$ of the cases showed mild and $\sim 40 \%$ severe peritumoral inflammation. In contrast, $20 \%$ of the tumors with distant metastasis showed severe inflammatory reaction. 

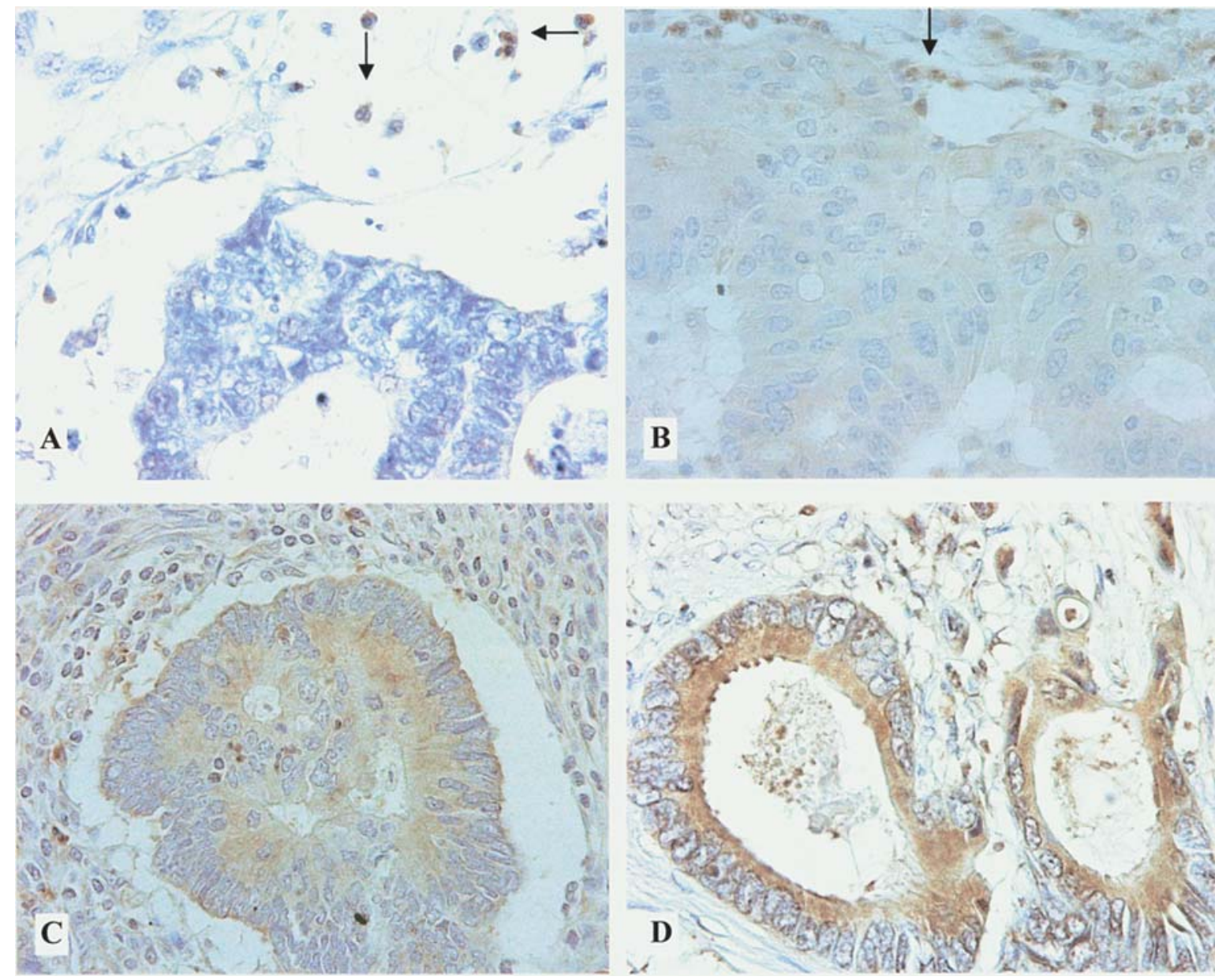

Figure 5. TLR4 expression profiles detected in CRC. (A) Specimen negative for TLR4. (B) Specimen characterized by a weak stain. (C) Example of a moderate stain. (D) Specimen showing a strong immunoreactivity. Positive cases showing a membranous and cytoplasmatic TLR4 localization. TLR4 detection in neutrophilic granulocytes was used as a positive internal control for the immunohistochemical reaction (marked with arrows). The sections were counterstained with Mayer's hematoxylin. Magnification, x400.

Table II. Correlation between the degree of peritumoral inflammation and TLR4 expression in non-metastatic (N0/M0), lymphogenous-metastatic $\left(\mathrm{N}^{+}\right)$and haematogenous-metastatic $\left(\mathrm{M}^{+}\right) \mathrm{CRC}$.

\begin{tabular}{lcccc}
\hline & \multicolumn{2}{c}{ Inflammation } & & \multicolumn{2}{c}{ TLR4 expression } \\
\cline { 2 - 3 } CRC & Degree & $\mathrm{n}$ & Positive & Negative \\
\hline N0/M0 & Mild & $23(57 \%)$ & $20(50 \%)$ & $3(7 \%)$ \\
& Severe & $17(43 \%)$ & $11(29 \%)$ & $6(14 \%)$ \\
$\mathrm{N}^{+}$ & Mild & $19(54 \%)$ & $11(31 \%)$ & $8(24 \%)$ \\
& Severe & $16(46 \%)$ & $12(33 \%)$ & $4(12 \%)$ \\
$\mathrm{M}^{+}$ & Mild & $32(80 \%)$ & $20(50 \%)$ & $12(30 \%)$ \\
& Severe & $8(20 \%)$ & $5(12 \%)$ & $3(8 \%)$ \\
\hline
\end{tabular}

Cases characterized by moderate and strong staining were defined as positive. Cases negative for TLR4 and showing weak TLR4 expression were defined as negative. $n$, number of investigated cases.

The ratio of mild and severe peritumoral inflammatory infiltration was 1.8 in both TLR4-positive and -negative tumors. Thus, a correlation between the degree of inflammation and the expression intensity of TLR 4 could not be postulated.

\section{Discussion}

Our previously published data showed that endothelial cells growing on conditioned media derived from LPS-stimulated 
CRC cell lines are characterized by upregulation of adhesion molecules such as ICAM-1, VCAM-1 and E-selectin $(8,9)$. Thus, we assumed that LPS stimulation results in induction of intracellular signaling pathways associated with regulation of expression of secretory molecules, among them cytokines, mediating the expression of the detected adhesion proteins. This observation is of great importance in the context of the essential role of the molecules mentioned above in the process of metastasis (29). Since CD14 and TLR4 have been reported as potent ligands for LPS in inflammatory cells, we focused our examination on their expression in eight CRC cell lines: SW837, HRT18, CX-1, CX-2, SW620, SW948, HT-29 and $\mathrm{CaCo} 2$; and in 115 surgical specimens derived from patients with non-metastatic and metastatic CRC $(10,11)$. Furthermore, we examined the influence of LPS stimulation on the expression of both receptors in question in the cell lines mentioned above. This analysis showed that under native conditions all cell lines were negative for CD14. With the exception of the well-differentiated $\mathrm{CaCo} 2$ cells, LPS stimulation did not influence their expression. Notably, LPS treatment of $\mathrm{CaCo} 2$ cells induced CD14 transcription. The translation of the transcript seemed, however, to be prevented by an as yet unknown mechanism which remains to be discovered.

Examination of CD14 expression in CRC revealed negative results similar to those detected in the aforementioned cell lines. This finding clearly suggests that CD14 cannot act as a mediator of LPS-induced signal transduction in both normal colorectal cells and tumors developed from them. As shown in this study CD14 expression in the latter was limited to the immuno-inflammatory cells of the stroma, whereas epithelial cells were definitely CD14 negative.

Our above findings on CD14 expression in CRC cell lines are consistent with the data published by Cario et al (19) for the human colon adenocarcinoma cell lines HT-29, CaCo2 and T84, and the mouse colon carcinoma CMT93 cells. They are, however, contradictory to the reports published by Funda et al (16) and McCracken et al (17) describing CD14 mRNA and protein expression in LPS-non-stimulated CRC cell lines among them HT-29 and $\mathrm{CaCo} 2$. Generally, the reports published on CD14 expression in CRC cells are inconsistent. Thus, speculation concerning the mode of action of LPS in these cells is not possible at present. The differences in culture conditions used by the various authors, mainly concerning the presence or absence of antibiotics, may be the source of these inconsistencies. As shown by Vega and De Maio (30), treatment with antibiotics appears to be principally responsible for reduction of the level of the CD14 receptor by internalization and accumulation within the endoplasmatic reticulum. This finding implies that successful determination of the native CD14 profiles in cultured cells requires cultivation in antibiotic-free media. Indeed, in the case of the positive epithelial CD14 expression reported by Funda et al and McCracken et al, the cells were cultured for several hours on antibiotic-free media. However, this point which is of obvious interest for the in vitro situation is not relevant in vivo. Our study concerning CD14 expression in colorectal tumors (115 cases) described here clearly shows that colon epithelia are CD14 negative whereas the peritumoral inflammatory cells are definitely positive. This finding suggests that in vivo in tumor epithelium LPS action cannot be mediated via the CD14 receptor.

As reported by Triantafilou and Triantafilou (11), LPS actions can also be mediated by molecules such as integrins, heat shock proteins, CXCR4, or CD55 molecules and the TLR4 receptor. The TLR4 receptor has been identified as an important component of mucosal immunity to LPS in acute and chronic inflammatory diseases $(10,11,19,24-26)$. As Cario and Podolsky (24) report, it is barely expressed in normal intestinal mucosa whereas it is strongly upregulated in patients suffering from ulcerative colitis and Crohn's disease. Furthermore, TLR4 has been found to be expressed in CRC cell lines such as HT-29, CaCo2 and T84 (19). Our results described here on TLR4 expression in various CRC cell lines support these previously reported data. Furthermore, the finding that LPS treatment of $\mathrm{CaCo} 2$ cells results in the upregulation of TLR4 expression generally supports findings suggesting its involvement in LPS-mediated cellular processes. Since, however, the expression of TLR4 levels in the other cell lines investigated: SW837, HRT18, CX-1, CX-2, SW620, SW948, and HT-29 was not influenced by this agent, we assume that LPS stimulation of TLR4 expression may be cell specific.

Studies concerning the expression of TLR4 in CRC have not yet been published. Thus, our studies on TLR4 expression in CRC implying its loss of expression to be associated with the metastatic potential of the tumor described here are of great importance in the context of the identification of the molecules regulating the metastatic potential of cells. The results presented clearly show that a progressive reduction of TLR4 expression correlates with an increase in the metastatic potential of the tumor. We suggest that the low levels of TLR4 expression in metastatic CRC may reflect the levels of hypoxia, which is a potent inducer of VEGF, one of the major angiogenic factors promoting tumor metastasis (31). Diminished TLR4 expression has been reported in endothelial cells under hypoxic conditions (32). Furthermore, this phenomenon may be associated with the immune tolerance of the tumor. The mechanisms of innate immune response to bacterial toxins differ in normal gut epithelium as compared to other tissues because of the contact with a high density of commensal bacteria. Here, immune-mediated signals downregulate the level of TLR4 expression. As a consequence the epithelial cells do not respond to LPS with an inflammatory reaction (33). We suggest that the lack of immune response in the tumor leading at least to tumor progression is maintained by the same molecules which are normally responsible for immune tolerance. Additionally, we hypothesize that loss of TLR4 is associated with lack of antitumoral immune response. Our hypothesis is consistent with studies proposing that signaling via TLR4 induces anticancer immunity through maturation of antigen-presenting cells and cytokine induction. Tsuji and colleagues (34) demonstrated that maturation of dendritic cells and cytokine induction by Mycobacterium bovis bacillus Calmette-Guerin, an anticancer immuno-adjuvant, are induced by TLR4. The intratumoral administration of bone narrow-derived dendritic cells after chemotherapy in combination with OK-432, a streptococcal immuno-potentiator in tumor-bearing mice, led to a significant infiltration of immune cells, to an increase in 
cytotoxic activities, and to an inhibition of tumor growth via TLR4 signaling (35). AILb-A, a protein extract of the parasitic plant Aeginetia indica L., induced potent antitumor immunity by TLR4-mediated pro-inflammatory cytokines (36).

However, observations contradicting the above findings are also published. Huang et al (37) describe that blocking of TLR4 signaling triggers the immune response by enhanced T-cell proliferation and natural killer cell activity. Generally, the controversial observations are due to the complexity of the relationships between inflammation, immunity and cancer. The inflammatory components in the tumor microenvironment have a dual role with respect to tumor behavior (6). They may either destroy neoplastic cells or potentiate tumor progression, depending on both the combination of the molecules and the level of their expression. In this context we do not observe an association of the expression intensity of TLR4 with the degree of peritumoral inflammation.

Notably, staining of TLR4 revealed not only membranous but also a cytoplasmatic distribution of this molecule. This observation is consistent with electron microscopic data described for T84 cells which showed that LPS stimulation leads to transcytotic trafficking of TLR4 and its redistribution from the apical to the basolateral side of the epithelial cells (38). At the present stage of our investigations the possibility that in colon carcinoma a similar strategic TLR4 redistribution directed from the luminal to the basal side of the epithelium takes place cannot be ruled out. This translocation could be responsible for TLR4-mediated communication between the epithelial cells and the lamina propria.

In summary, our data suggest that CD14 is not relevant to CRC in the context of LPS action. On the other hand, TLR4 action seems to be important in the context of metastasis of these cancers. Reduced expression of TLR4 strongly correlates with increase of the metastatic potential of the tumor cells. Which cellular mechanisms associated with the metastatic process are particularly driven by downregulation of TLR4 still have to be elucidated.

\section{References}

1. Parkin DM, Bray F, Ferlay J and Pisani P: Global cancer statistics, 2002. CA Cancer J Clin 55: 74-108, 2005.

2. Mareel MM, Van Roy FM and Bracke ME: How and when do tumor cells metastasize? Crit Rev Oncog 4: 559-594, 1993.

3. Mantovani A, Bussolino F and Introna M: Cytokine regulation of endothelial cell function: from molecular level to the bedside. Immunol Today 18: 231-240, 1997.

4. Balkwill $\mathrm{F}$ and Mantovani A: Inflammation and cancer: back to Virchow? Lancet 357: 539-545, 2001.

5. Coussens LM and Werb Z: Inflammation and cancer. Nature 420: 860-867, 2002.

6. Wilson J and Balkwill F: The role of cytokines in the epithelial cancer microenvironment. Semin Cancer Biol 12: 113-120, 2002.

7. Schletter J, Heine H, Ulmer AJ and Rietschel ET: Molecular mechanisms of endotoxin activity. Arch Microbiol 164: 383-389, 1995.

8. Simiantonaki N, Jayasinghe C and Kirkpatrick CJ: Effect of pro-inflammatory stimuli on tumor cell-mediated induction of endothelial cell adhesion molecules in vitro. Exp Mol Pathol 73: 46-53, 2002.

9. Simiantonaki N, Jayasinghe C and Kirkpatrick CJ: Differential endothelial CAM-expression after stimulation with supernatants of LPS- and cytokine-stimulated HT-29 and ST-ML-12 tumor cells growing as monolayer cultures and multicellular spheroids. Anticancer Res 22: 2641-2649, 2002.
10. Triantafilou $\mathrm{M}$ and Triantafilou K: Lipopolysaccharide recognition: CD14, TLRs and the LPS-activation cluster. Trends Immunol 23: 301-304, 2002.

11. Triantafilou $\mathbf{M}$ and Triantafilou $\mathrm{K}$ : The dynamics of LPS recognition: complex orchestration of multiple receptors. J Endotoxin Res 11: 5-11 2005.

12. Wright SD, Ramos RA, Tobias PS, Ulevitch RJ and Mathison JC: CD14, a receptor for complexes of lipopolysaccharide (LPS) and LPS binding protein. Science 249: 1431-1433, 1990.

13. Ziegler-Heitbrock HW and Ulevitch RJ: CD14: cell surface receptor and differentiation marker. Immunol Today 14: 121-125, 1993.

14. Sugawara S, Sugiyama A, Nemoto E, Rikiishi H and Takada H: Heterogeneous expression and release of CD14 by human gingival fibroblasts: characterization and CD14-mediated interleukin-8 secretion in response to lipopolysaccharide. Infect Immun 66: 3043-3049, 1998.

15. Jersmann HP, Hii CS, Hodge GL and Ferrante A: Synthesis and surface expression of CD14 by human endothelial cells. Infect Immun 69: 479-485, 2001.

16. Funda DP, Tuckova L, Farre MA, Iwase T, Moro I and Tlaskalova-Hogenova H: CD14 is expressed and released as soluble CD14 by human intestinal epithelial cells in vitro: lipopolysaccharide activation of epithelial cells revisited. Infect Immun 69: 3772-3781, 2001.

17. McCracken VJ, Chun T, Baldeon ME, Ahrne S, Molin G, Mackie RI and Gaskins HR: TNF-alpha sensitizes HT-29 colonic epithelial cells to intestinal lactobacilli. Exp Biol Med 227: 665-670, 2002 .

18. Yoshioka T, Morimoto Y, Iwagaki H, Itoh H, Saito S, Kobayashi N, Yagi T and Tanaka N: Bacterial lipopolysaccharide induces transforming growth factor beta and hepatocyte growth factor through toll-like receptor 2 in cultured human colon cancer cells. J Int Med Res 29: 409-420, 2001.

19. Cario E, Rosenberg IM, Brandwein SL, Beck PL, Reinecker HC and Podolsky DK: Lipopolysaccharide activates distinct signaling pathways in intestinal epithelial cell lines expressing Toll-like receptors. J Immunol 164: 966-972, 2000.

20. Barnes PJ and Karin M: Nuclear factor-kappaB: a pivotal transcription factor in chronic inflammatory diseases. $\mathrm{N}$ Engl J Med 336: 1066-1071, 1997.

21. Okamoto M, Oshikawa T, Ohe G, Nishikawa H, Furuichi S, Tano T, Moriya Y, Saito M and Sato M: Severe impairment of anti-cancer effect of lipoteichoic acid-related molecule isolated from a penicillin-killed streptococcus pyogenes in toll-like receptor 4-deficient mice. Int Immunopharmacol 1: 1789-1795, 2001 .

22. Muzio M, Bosisio D, Polentarutti N, D'amico G, Stoppacciaro A, Mancinelli R, van't Veer C, Penton-Rol G, Ruco LP, Allavena P and Mantovani A: Differential expression and regulation of toll-like receptors (TLR) in human leukocytes: selective expression of TLR3 in dendritic cells. J Immunol 164: 5998-6004, 2000.

23. Faure E, Thomas L, Xu H, Medvedev A, Equils O and Arditi M: Bacterial lipopolysaccharide and IFN-gamma induce Toll-like receptor 2 and Toll-like receptor 4 expression in human endothelial cells: role of NF-kappa B activation. J Immunol 166: 2018-2024, 2001.

24. Cario E and Podolsky DK: Differential alteration in intestinal epithelial cell expression of toll-like receptor 3 (TLR3) and TLR4 in inflammatory bowel disease. Infect Immun 68: 70107017, 2000.

25. Ortega-Cava CF, Ishihara S, Rumi MA, Kawashima K, Ishimura N, Kazumori H, Udagawa J, Kadowaki Y and Kinoshita Y: Strategic compartmentalization of Toll-like receptor 4 in the mouse gut. J Immunol 170: 3977-3985, 2003.

26. Bocker U, Yezerskyy O, Feick P, Manigold T, Panja A, Kalina U, Herweck F, Rossol S and Singer MV: Responsiveness of intestinal epithelial cell lines to lipopolysaccharide is correlated with Toll-like receptor 4 but not Toll-like receptor 2 or CD14 expression. Int J Colorectal Dis 18: 25-32, 2003.

27. Schwende H, Fitzke E, Ambs P and Dieter P: Differences in the state of differentiation of THP-1 cells induced by phorbol ester and 1,25-dihydroxyvitamin D3. J Leukoc Biol 59: 555-561, 1996.

28. International Union Against Cancer (UICC): TNM Classification of Malignant Tumors. Sobin LH and Wittekind C (eds). 5th edition, 1997.

29. Zetter BR: Adhesion molecules in tumor metastasis. Semin Cancer Biol 4: 219-229, 1993. 
30. Vega VL and De Maio A: Geldanamycin treatment ameliorates the response to LPS in murine macrophages by decreasing CD14 surface expression. Mol Biol Cell 14: 764-773, 2003.

31. Ferrara N: Vascular endothelial growth factor: basic science and clinical progress. Endocr Rev 25: 581-611, 2004.

32. Ishida I, Kubo H, Suzuki S, Suzuki T, Akashi S, Inoue K, Maeda S, Kikuchi H, Sasaki H and Kondo T: Hypoxia diminishes toll-like receptor 4 expression through reactive oxygen species generated by mitochondria in endothelial cells. J Immunol 169: 2069-2075, 2002.

33. Abreu MT, Vora P, Faure E, Thomas LS, Arnold ET and Arditi M: Decreased expression of Toll-like receptor-4 and MD-2 correlates with intestinal epithelial cell protection against dysregulated proinflammatory gene expression in response to bacterial lipopolysaccharide. J Immunol 167: 1609-1616, 2001.

34. Tsuji S, Matsumoto M, Takeuchi O, Akira S, Azuma I, Hayashi A, Toyoshima K and Seya T: Maturation of human dendritic cells by cell wall skeleton of Mycobacterium bovis bacillus CalmetteGuerin: involvement of toll-like receptors. Infect Immun 68: 6883-6890, 2000.
35. Ahmed SU, Okamoto M, Oshikawa T, Tano T, Sasai A, Kan S, Hiroshima T, Ohue H, Moriya Y, Ryoma Y, Saito M and Sato M: Anti-tumor effect of an intratumoral administration of dendritic cells in combination with TS-1, an oral fluoropyrimidine anti-cancer drug, and OK-432, a streptococcal immunopotentiator: involvement of toll-like receptor 4 . J Immunother 27: 432-441, 2004.

36. Okamoto M, Oh-E G, Oshikawa T, Furuichi S, Tano T, Ahmed SU, Akashi S, Miyake K, Takeuchi O, Akira S, Himeno K, Sato M and Ohkubo S: Toll-like receptor 4 mediates the antitumor host response induced by a 55-kilodalton protein isolated from Aeginetia indica L., a parasitic plant. Clin Diagn Lab Immunol 11: 483-495, 2004.

37. Huang B, Zhao J, Li H, He KL, Chen Y, Chen SH, Mayer L, Unkeless JC and Xiong H: Toll-like receptors on tumor cells facilitate evasion of immune surveillance. Cancer Res 65: 5009-5014, 2005.

38. Cario E, Brown D, McKee M, Lynch-Devaney K, Gerken G and Podolsky DK: Commersal-associated molecular patterns induce selective toll-like receptor-trafficking from apical membrane to cytoplasmatic compartments in polarized intestinal epithelium. Am J Pathol 160: 165-173, 2002. 